\section{OPEN ACCESS}

Edited by: Marcello Locatelli, Università degli Studi "G. d'Annunzio"

Chieti-Pescara, Italy

Reviewed by:

Claudio Ferrante, Università degli Studi "G. d'Annunzio"

Chieti-Pescara, Italy

Gokhan Zengin,

Selçuk University, Turkey

*Correspondence:

Yanjun Zhang

tianjin_tcm001@sina.com

tThese authors have contributed equally to this work and should be co-first authors.

Specialty section:

This article was submitted to

Ethnopharmacology,

a section of the journal Frontiers in Pharmacology

Received: 21 March 2018 Accepted: 18 May 2018

Published: 19 June 2018

Citation:

Li Y, Li Y, LuW, Li H, Wang Y, LuO H, Wu Y, Dong W, Bai $G$ and Zhang Y (2018) Integrated Network Pharmacology and Metabolomics Analysis of the Therapeutic Effects of Zi Dian Fang on Immune Thrombocytopenic Purpura. Front. Pharmacol. 9:597. doi: 10.3389/fphar.2018.00597

\title{
Integrated Network Pharmacology and Metabolomics Analysis of the Therapeutic Effects of Zi Dian Fang on Immune Thrombocytopenic Purpura
}

\begin{abstract}
Yubo $\mathrm{Li}^{1+}$, Yamei Li't, Wenliang Lu², Hongbin $\mathrm{Li}^{2}$, Yuming Wang ${ }^{1}$, Houmin Luo', Yuanyuan Wu ${ }^{1}$, Wenying Dong ${ }^{1}$, Gang Bai ${ }^{3}$ and Yanjun Zhang ${ }^{1 *}$
\end{abstract}

1 Tianjin State Key Laboratory of Modern Chinese Medicine, School of Traditional Chinese Materia Medica, Tianjin University of Traditional Chinese Medicine, Tianjin, China, ${ }^{2}$ Tasly Institute, Tasly Pharmaceutical Group, Tianjin, China, ${ }^{3}$ College of Pharmacy, Nankai University, Tianjin, China

Current hormone-based treatments for immune thrombocytopenic purpura (ITP) are associated with potentially serious adverse reactions. Zi Dian Fang (ZDF) is a multitarget Traditional Chinese Medicine (TCM) used to treat both the symptoms and root causes of ITP, with fewer side effects than hormone-based treatments. This study analysis of the therapeutic effects of ZDF on ITP from three aspects: platelet proliferation, immunoregulation, and inflammation. After detection of 52 chemical constituents of ZDF by UPLC-Q-TOF/MS, The main targets and pathways affected by ZDF were screened by network pharmacology and verified by Western blot and ELISA. Meanwhile, metabolomics analysis were applied to a mouse model of ITP to identify and screen endogenous terminal metabolites differentially regulated by ZDF. Integrated network pharmacology and metabolomics analysis of the therapeutic effects of ZDF on ITP may be as follows: ZDF counteracts ITP symptoms mainly by inhibiting Ras/MAPKs (Ras/Mitogen-activated protein kinases) pathway, and the expression of upstream protein (Ras) and downstream protein ( $\mathrm{p}$-ERK, p-JNK, p-p38) were inhibited, which affects the content of effect index associated with proliferation (Thrombopoietin, TPO; Granulocyte-macrophage colony stimulating factor, GM-CSF), inflammation (Tumor necrosis factor- $\alpha$, TNF- $\alpha$; Interleukin-6, IL-6), immune (Interleukin-2, IL-2; Interferon-gamma, IFN- $\gamma$; Interleukin-4, IL-4), so that the body's arginine, $\Delta^{12}$ prostaglandin j2 ( $\Delta^{12}-\mathrm{PGJ} 2$ ), 9-cis-Retinoic Acid, sphingosine-1-phosphate (S1P), oleic acid amide and other 12 endogenous metabolites significantly changes. Considering the established safety profile, the present study suggests ZDF may be a useful alternative to hormone-based therapies for the treatment of ITP.

Keywords: network pharmacology, metabonomics, biomarkers, immune thrombocytopenic purpura, mechanism

\section{INTRODUCTION}

Immune thrombocytopenic purpura (ITP) is an autoimmune vasculitis characterized by skin, mucosal and/or visceral bleeding resulting from autoantibody-mediated platelet damage (Rodeghiero et al., 2009). ITP is a relatively common hemorrhagic disease in children. While acute ITP typically resolves within 3 months, chronic refractory ITP has characteristic long disease course 
(Swain et al., 2016; Queliza et al., 2017). Adrenocortical hormone therapy (prednisone acetate, cortisone, etc.) is often used for the clinical treatment of ITP, but is associated with adverse reactions, and long-term use seriously compromises the body's defense system (Lembke et al., 2013; Arnett et al., 2016). Traditional Chinese Medicine (TCM) offers a holistic approach to patient treatment and care, viewing disease as the result of an internal imbalance between the body's energy (Yang) and substance (Yin). Because TCM aims to restore homeostasis basically by normalizing immune and metabolic functions, multi-ingredient TCM formulae are particularly suited for the treatment of complex diseases. Naturally sourced TCM preparations have been used, and their safety tested, over 1000s of years in East Asian countries. However, lack of characterization of the biologically active principles in TCM herbal formulations and lack of evidence-based standards of quality and efficacy have hampered their acceptance and use in other countries (Zhou et al., 2014).

$\mathrm{Zi}$ Dian Fang (ZDF) is a TCM formula composed of Hedysarum multijugum Maxim (zhihuangqi in Chinese, ZHQ), Cortex Moutan (mudanpi in Chinese, MDP), Fructus Ligustri Lucidi (nvzhenzi in Chinese, NZZ), Ecliptae Herba (mohanlian in Chinese, MHL), Sweet potato vine (fanshuteng, FST), Donkey-hide gelatin (ejiao in Chinese, AJ), Herba Selaginellae Moellendorffii (juanbai in Chinese, JB), Panax notoginseng (sanqi in Chinese, SQ), Peanut skin (huashengyi in Chinese, HSY), and Radix liquiritiae (gancao in Chinese, GC). According to TCM precepts, this herbal decoction is used to treat ITP by 'benefiting qi' (vital energy/flow) and 'nourishing yin,' i.e., 'cooling the blood' and favoring hemostasis. However, its chemical composition and the molecular basis of its therapeutic effects on ITP have not been fully studied (Han et al., 2014; Wagner et al., 2016).

Complex diseases are often caused by the accumulation of small defects in many genes, rather than large defects in a few ones; therefore, treatments aimed at single molecular targets frequently do not work (Falvo et al., 2013). In 2007, Andrew L. Hopkins introduced the concept of "Network pharmacology," its core idea being to identify the biological networks that interconnect disease features, bioactive agents, and drug targets with the goal of optimizing treatment strategies. Interestingly, the core idea of network pharmacology coincides with the tenet of overall regulation of TCM (Hopkins, 2008). Thus, network

\footnotetext{
Abbreviations: $\Delta^{12}$-PGJ2, $\Delta^{12}$-prostaglandin J2; AA, arachidonic acid; ANOVA, analysis of variance; COX, cyclooxygenase; ELISA, enzyme-linked immunosorbent assay; EPA, eicosapentaenoic acid; ERK, extracellular-signal regulated protein kinase; ESI, electrospray ionization; GM-CSF, Granulocyte-macrophage colony stimulating factor; GP-APS, guinea pig anti-mouse platelet serum; HMDB, Human Metabolome Database; HPA, hypothalamic-pituitary-adrenal; HRP, horseradish peroxidase; IFN- $\gamma$, interferon gamma; IL-2, interleukin-2; IL-4, interleukin-4; IL6, interleukin-6; ITP, immune thrombocytopenic purpura; iNOS, inducible nitric oxide synthase; JNK, c-Jun N-terminal kinase; KEGG, Kyoto Encyclopedia of Genes and Genomes; LOX, lipoxyoxygenase; MAPK, mitogen-activated protein kinase; NO, nitric oxide; NOS, nitric oxide synthase; p38MAPK, p38 mitogenactivated protein kinase; PCA, principal component analysis; PGI2, prostacyclin; PLS-DA, partial least squares-discriminant analysis; PVDF, Polyvinylidene fluoride; QC, quality control; SDS-PAGE, Sodium dodecyl sulfate-polyacrylamide gel electrophoresis; S1P, Sphingosine 1-phosphate; TCM, traditional chinese medicine; Th1, T helper 1; Th2, T helper 2; TNF- $\alpha$, tumor necrosis factor alpha; TPO, Thrombopoietin; TXA2, thromboxane A2; VIP, Variable Importance Plot; ZDF, Zi Dian Fang.
}

pharmacology analysis has been applied to the study of TCM formulae to predict the molecular targets and signaling pathways affected by TCM prescriptions for different diseases (Chen et al., 2015; Mao et al., 2017). Prescription compatibility, which refers to the structural composition of herbal medicines, is flexible and complex, as elimination of the original substance, generation of new substances, or changes in the contents of active ingredients may occur in the process of boiling and extraction. However, network pharmacology can only be used to forecast targets and pathways affected by individual chemical components; it can not explain changes in metabolites that occur in the body after taking the medicine, so it requires cellular and/or animal experimental verification and further support of 'omics' technologies such as chemomics and metabolomics (Pang et al., 2018).

The metabolomics technology emerges with the development of systems biology, and is an important part of it. It takes the whole organism as the research object, studies the effects of drugs and other factors on terminal metabolites by observing the changes that result after stimulating or disturbing the biological system, and then explains drugs actions at the metabolic level (Shajahan-Haq et al., 2015; Ju et al., 2016). Thus, the combination of network pharmacology and metabolomics allows identifying and connecting biologically active substances, molecular targets, and metabolic effects and is thus ideally suited to study the mechanistic basis of TCM treatments.

In this study, we first identified the chemical constituents of ZDF by UPLC-Q-TOF/MS, a highly sensitive and specific technique (Mejías et al., 2014). Next, we applied network pharmacology methods to generate an ITP "chemical composition-target-pathway" regulatory network centered on three main aspects: platelet proliferation, immunity, and inflammation. Subsequently, we used an ITP mouse model and metabolomics analysis to verify ZDF-induced alterations in the major predicted pathway, i.e., the Ras/MAPKs signaling pathway, which includes Ras and its main downstream targets: ERK, JNK, and p38MAPK. Finally, through integrative analysis of network pharmacology and metabolomics data we constructed a herb-chemical constituent-targets-pathwayregulatory index-metabolite regulatory network representation of ZDF's pharmacological activity against ITP. By characterizing the ZDF composition and its therapeutic mechanisms on ITP we lay the foundation for the clinical application of this TCM prescription.

\section{MATERIALS AND METHODS}

\section{Instruments and Reagents}

HPLC analysis was performed in a Waters UPLC-Q-TOF-MS system (Waters, United States). ACQUITY UPLC BEH C18 columns $(2.1 \times 100 \mathrm{~mm}, 1.7 \mu \mathrm{m}$, Waters $)$, and ACQUITY UPLC HSS C18 columns $(2.1 \times 100 \mathrm{~mm}, 1.8 \mu \mathrm{m}$, Waters $)$ were purchased from Waters, United States. Complete Freund's adjuvant and incomplete Freund's adjuvant were from Sigma, United States. Antibodies against p38, phospho-p38 (Thr 180/Tyr 182), p44/42 (ERK1/2), phospho-p44/42 (Thr 202/Tyr 204), SAPK/JNK, and phospho-SAPK/JNK (Thr 183/Tyr 185) 
were from Cell Signaling Technology, United States. Alkaline phosphatase-labeled goat anti-rabbit Ig G, RIPA lysis buffer, PMSF protease inhibitor, and protein phosphatase inhibitor mixture were purchased from Solarbio Co., Ltd. (Beijing, China). Mouse serum GM-CSF, TPO, IL-2, IL-4, IFN- $\gamma$, TNF$\alpha$, and IL-6 ELISA kits were purchased from Baoman Co., Ltd. (Shanghai, China). The BCA Protein Quantification Kit was purchased from Yupinghao Biological Technology Co., Ltd. (Tianjin, China). Purified water was purchased from Wahaha Company (Hangzhou, China). Prednisone acetate tablets were purchased from Lisheng Pharmaceutical Co., Ltd. (Tianjin, China). Hedysarum multijugum Maxim, Cortex Moutan, Fructus Ligustri Lucidi, Ecliptae Herba, Sweet potato vine, Donkey-hide gelatin, Herba Selaginellae Moellendorffi, Panax notoginseng, Peanut skin, Radix liquiritiae were purchased from Tong Ren Tang Co., Ltd. (Beijing, China).

\section{Preparation of Zi Dian Fang Test Solution}

Ten medicinal herbs were used to prepare the ZDF test solution: ZHQ (25 g), MDP (10 g), NZZ (10 g), MHL (10 g), and SQ $(3.35 \mathrm{~g})$ were extracted three times with 8 times the amount of $70 \%$ ethanol under reflux for $60 \mathrm{~min}$, following by filtering. FST (16.65 g), JB (10 g), HSY (5 g), and GC (5 g) were extracted three times with 8 times the amount of pure water under reflux for $60 \mathrm{~min}$. Then the extracted solutions were filtered and combined with the alcohol extract. AJ (5 g) was mixed with the liquid medicine to prepare the ZDF test solution. Ten kinds of single medicinal herbs were extracted, collected and prepared according to the formula in accordance with the proportion of single herbs for the test solution.

\section{UPLC-Q-TOF/MS Analysis}

\section{Chromatographic Conditions}

ACQUITY UPLC BEH C18 columns $(2.1 \times 100 \mathrm{~mm}, 1.7 \mu \mathrm{m}$, Waters); flow rate, $0.3 \mathrm{~mL} / \mathrm{min}$; column temperature, $30^{\circ} \mathrm{C}$; injection volume, $5 \mu \mathrm{L}$. Mobile phases: A phase, $0.1 \%$ formic acid solution; B phase, acetonitrile. Specific elution procedures were as follows: $0-1 \mathrm{~min}, \mathrm{~A}: 99-99 \%$; 1-10 $\mathrm{min}, \mathrm{A}: 99-60 \%$; 10-20 $\mathrm{min}$, A: 60-30\%; 30-31 min, A: 1-1\%; 31-40 min, A: 1-99\%.

\section{Mass Spectrometry Conditions}

Mass spectral analyses were carried out in positive ionization mode using ESI. Drying gas temperature, $350^{\circ} \mathrm{C}$; drying gas flow, $10 \mathrm{~mL} / \mathrm{min}$; desolvation gas flow, $600 \mathrm{~L} / \mathrm{h}$; nebulizer pressure, 310 psi; range of data acquisition, $100-1500 \mathrm{Da}$.

\section{Data Analysis}

Based on literature reports of herbs' chemical composition, peak alignment and peak match lists were generated by MassLynx software, and the retention time, precise mass number, and secondary fragment information for each chemical component were obtained. Then, putative chemical categories and fragmentation rules of mass spectra were summarized. Finally, we compared the literature and standard information to confirm the chemical composition of ZDF. We obtained the total ion flow chart for single herbs, compared it with the total ion chromatogram of ZDF, and determined the ascription of each chemical component.

\section{Network Pharmacology Analysis Construction of Zi Dian Fang "Chemical Constituent-Target-Pathway" Regulatory Network}

Chemical constituents identified by UPLC-Q-TOF/MS were used as the research object. First, 3D structures were drawn using Chem Bio Office 2010 software and stored in sdf format. The Pharm Mapper server was used for potential target prediction analysis. The top 10 targets with high matching degrees were introduced into the Uni Prot database ${ }^{1}$ to obtain their official names for subsequent pathway analysis. Target information was imported into the MAS 3.0 database, and relevant pathway information was obtained. We analyzed the pathways using the gene analysis function stored in the KEGG database. Finally, Cytoscape 2.6.0 software was used to construct the "chemical composition-target-pathway" regulatory network of ZDF for ITP treatment.

\section{Verification of Network Pharmacology-Based Virtual Screening Results}

Animals

SPF-grade healthy BALB/c mice (8 weeks old, weighing $20 \pm 2$ g) were purchased from Beijing Weitong Lihua Technology Co. Ltd. under license number "SCXK (Jing) 2012-0001." Ordinary level guinea pigs were purchased from Beijing Weitong Lihua Technology Co. Ltd. under license number "SCXK (Jing) 20120015." The growth environment consisted of 12-h day/night cycles, ambient temperature was $23 \pm 2{ }^{\circ} \mathrm{C}$, with humidity of $35 \pm 5 \%$. All experiments were carried out in accordance with Chinese national laws and local guidelines. The animal study was approved by the Animal Ethics Committee of Tianjin University of Traditional Chinese Medicine (approval number TCM-2012078F01).

\section{Preparation of Guinea Pig Anti-mouse Platelet Serum (GP-APS)}

\section{Antigen preparation}

Mouse blood obtained by retro-orbital bleeding was mixed with EDTA-Na $\mathrm{Na}_{2}$ anticoagulant, and platelets separated by gradient centrifugation. Samples were then diluted with saline solution and after microscopic observation, their concentration was adjusted to $1 \sim 2 \times 10^{9} / \mathrm{L}$.

\section{Immunological methods}

Platelet suspensions were diluted 1:1 in incomplete Freund's adjuvant, or in complete Freund's adjuvant (water-in-oil emulsion). Complete Freund's adjuvant antigen was injected subcutaneously into guinea pigs' foot, palm, and back, $(100 \mu \mathrm{L}$ per injection site) at 0 weeks. Antigens in incomplete Freund's adjuvant were subcutaneously injected into the same anatomical locations at 1,2, and 4 weeks. At week 5, blood was drawn from the guinea pigs' hearts and let to coagulate at room temperature for $1 \mathrm{~h}$. Blood samples were placed at $4^{\circ} \mathrm{C}$ overnight, centrifuged

\footnotetext{
${ }^{1}$ http://www.uniprot.org/
} 
at 2,000 rpm $\times 20 \mathrm{~min}$ and the supernatant, consisting of GP-APS was placed at $-20^{\circ} \mathrm{C}$ until use (Zhang et al., 2016).

\section{Antiserum treatment}

GP-APS was removed from the freezer, thawed, and placed in a water bath at $56^{\circ} \mathrm{C}$ for $30 \mathrm{~min}$. After adsorption with the same amount of BALB/c mouse red blood cells, GP-APS was diluted 1:4 (v:v) with saline (Zhang et al., 2016).

\section{In Vivo ITP Model and Treatment}

Sixty mice were randomly divided into six groups (10 mice/group): (1) control; (2) model control; (3) low-dose ZDF treatment; (4) middle-dose ZDF treatment; (5) high-dose ZDF treatment; and (6) prednisone acetate treatment. Except for the control group, which received saline, the other groups were injected intraperitoneally with $1: 4$ (v:v) GP-APS at 0 , $1,3,5,7,9,11$, and 13 days to model ITP. ZDF was administered to the low-, middle-, and high-treatment groups. Prednisone acetate was administered to prednisone acetate treatment group. Twenty-four h after the last GP-APS injection, the mice were given intragastric administration once a day for 14 consecutive days, and the animals were sacrificed on the 15th day after sampling (Yang et al., 1994). Details are shown in Table 1.

\section{Sample Collection}

After 7 and 14 days of ITP modeling, peripheral blood platelet numbers were measured using an automatic blood cell analyzer. After 14 days of modeling, blood was taken without anticoagulation by retro-orbital bleeding, and centrifuged at 3,500 rpm for $10 \mathrm{~min}$ to separate the serum, which was stored at $-80^{\circ} \mathrm{C}$ for metabolic studies. On day 15 , mice were sacrificed and the spleens were dissected, weighed, and quickly transferred to a $-80^{\circ} \mathrm{C}$ freezer, for target protein detection. The right femurs were stripped, their ends cut off, and the bone marrow was repeatedly flushed out into a $1.5 \mathrm{~mL}$ sample tube using a small amount of saline. After centrifugation at $2,000 \mathrm{rpm}$ for $10 \mathrm{~min}$, the supernatant was discarded and a smear of the lower layer of bone marrow cells was fixed in methanol, stained in Swiss-Giemsa, and sealed with neutral gum for bone marrow megakaryocyte count.

\section{Western Blot Analysis}

Spleen tissue was homogenized, and equal amounts of protein (quantified by the BCA method) were separated by $12 \%$ SDSPAGE, transferred to a PVDF membrane, and then blocked with $5 \%$ BSA. Primary antibodies were incubated overnight, followed by TBST washing and incubation with secondary antibodies for $1 \mathrm{~h}$. After washing, $200 \mu \mathrm{l}$ of luminol were added for UVP chemiluminescence detection.

\section{ELISA Analysis}

The levels of serum cytokines (IL-2, IFN- $\gamma$, TPO, GM-CSF, TNF- $\alpha$, and IL-6) were detected by ELISA kit according to the manufacturer's instructions. The kit was removed from the refrigerator at $4^{\circ} \mathrm{C}$ centigrade and balanced at room temperature for $20 \mathrm{~min}$. Standard wells, sample wells and blank wells were set. Blank hole as a reference. Standard wells were added to different concentration of standard $50 \mu \mathrm{L}$. Sample wells were added to the sample diluent $40 \mu \mathrm{L}$, after adding the sample to be tested $10 \mu \mathrm{L}$. Subsequently, the standard wells and sample wells were added to the HRP labeled detection antibody $100 \mu \mathrm{L}$. The wells were sealed with a sealing film and incubated at $37^{\circ} \mathrm{C}$ for $60 \mathrm{~min}$. Then discard the liquid, added washing liquid and repeated washing five times. After the liquid was dried, all the wells were added to the substrate $\mathrm{A}$ and $\mathrm{B}$ each $50 \mu \mathrm{L}$, and incubated at $37^{\circ} \mathrm{C}$ for $15 \mathrm{~min}$ protect form light. Subsequently, $50 \mu \mathrm{L}$ of the stop solutions were added to all the wells, and the absorbance (OD) value of each well was measured at a wavelength of $450 \mathrm{~nm}$. According to the OD value and concentration of the standard wells, obtained the standard linear regression equation of each enzyme, and calculated the concentration of each sample hole. Based on the concentration of each sample well, the average value of the contents of each group of related enzymes, the change in content and the significance with respect to the control group were calculated.

\section{Metabolomics Analysis}

\section{Sample Preparation}

Following thawing at room temperature, $100 \mu \mathrm{l}$ aliquots of serum samples were mixed with $300 \mu \mathrm{l}$ of acetonitrile, sonicated for $10 \mathrm{~min}$ on ice, centrifuged at $13,000 \mathrm{rpm}$ for $15 \mathrm{~min}$ at $4^{\circ} \mathrm{C}$, and the supernatants analyzed by UPLC-Q-TOF/MS.

\section{QC Sample Preparation}

To ensure the stability and repeatability of the systems, plasma samples singled out from each group were pooled as a quality control (QC) sample, mixed for $1 \mathrm{~min}$, and centrifuged at $13,000 \mathrm{rpm}$ for $10 \mathrm{~min}$ to obtain supernatants.

\section{UPLC-Q-TOF/MS Serum Analysis Chromatographic Conditions}

ACQUITY UPLC HSS C18 columns $(2.1 \times 100 \mathrm{~mm}, 1.8 \mu \mathrm{m}$, Waters); flow rate, $0.3 \mathrm{~mL} / \mathrm{min}$; column temperature, $40^{\circ} \mathrm{C}$; injection volume, $5 \mu \mathrm{L}$. For the gradient elution process, the $\mathrm{A}$ phase was $0.1 \%$ formic acid/water, the B-phase was $0.1 \%$ formic acid/acetonitrile. The gradient started with $99 \%$ A, followed by 0-0.5 min, A: 99 to $99 \%$; $0.5-2 \mathrm{~min}, \mathrm{~A}: 99$ to $50 \%$; 2 to $9 \mathrm{~min}, \mathrm{~A}$ : 50 to $1 \%$; $9-10 \mathrm{~min}, \mathrm{~A}: 1$ to $1 \%$; $10-10.5 \mathrm{~min}, \mathrm{~A}: 1$ to $99 \%$; and 10.5-12 min, A: 99 to $99 \%$.

\section{MS Conditions}

Using ESI as source, mass spectral analyses were carried out in positive ionization mode. High-purity $\mathrm{N}_{2}$ was used as auxiliary ESI gas for solvent removal: drying gas flow, $10 \mathrm{ml} / \mathrm{min} ; \mathrm{N}_{2}$ temperature, $325^{\circ} \mathrm{C}$; pressure of atomized gas was $350 \mathrm{psi}$; desolvation gas flow, $600 \mathrm{~L} / \mathrm{h}$; capillary voltage, $3.5 \mathrm{kV}$; data acquisition range was 50-1000 Da.

\section{Methodological Validation}

For instrument precision testing, the same QC sample solutions were continuously injected 6 times, and 20 chromatographic peaks were randomly selected. Relative 
TABLE 1 | Animal experiment grouping and dosing regimen.

\begin{tabular}{|c|c|c|c|c|c|}
\hline Grouping & Number & Drug & Dose & Mode of administration & Sampling time (day) \\
\hline \multirow[t]{2}{*}{ Control group } & 10 & Normal saline & 5 mL/kg/day & i.p., successive administration & 8 \\
\hline & & Distilled water & $10 \mathrm{~mL} / \mathrm{kg} /$ day & i.g., successive administration & 14 \\
\hline \multirow[t]{2}{*}{ Model group } & 10 & GP-APS & $5 \mathrm{~mL} / \mathrm{kg} /$ day & i.p., next day administration & 8 \\
\hline & & Distilled water & $10 \mathrm{~mL} / \mathrm{kg} /$ day & i.g., successive administration & 14 \\
\hline \multirow[t]{2}{*}{ ZDF high dose group } & 10 & GP-APS & $5 \mathrm{~mL} / \mathrm{kg} /$ day & i.p., next day administration & 8 \\
\hline & & ZDF-H & $16.8 \mathrm{~g} / \mathrm{kg} /$ day & i.g., successive administration & 14 \\
\hline \multirow[t]{2}{*}{ ZDF middle dose group } & 10 & GP-APS & $5 \mathrm{~mL} / \mathrm{kg} /$ day & i.p., next day administration & 8 \\
\hline & & ZDF-M & $8.4 \mathrm{~g} / \mathrm{kg} /$ day & i.g., successive administration & 14 \\
\hline \multirow[t]{2}{*}{ ZDF low dose group } & 10 & GP-APS & $5 \mathrm{~mL} / \mathrm{kg} /$ day & i.p., next day administration & 8 \\
\hline & & ZDF-L & $4.2 \mathrm{~g} / \mathrm{kg} /$ day & i.g., successive administration & 14 \\
\hline \multirow[t]{2}{*}{ Prednisone acetate group } & 10 & GP-APS & $5 \mathrm{~mL} / \mathrm{kg} /$ day & i.p., next day administration & 8 \\
\hline & & Prednisone acetate & $7.8 \mathrm{mg} / \mathrm{kg} / \mathrm{day}$ & i.g., successive administration & 14 \\
\hline
\end{tabular}

i.p., intraperitoneal injection; i.g., oral administration by gavage.

standard deviation (RSD) values of peak area and retention time of the 20 chromatographic peaks were calculated.

For method precision testing, 6 QC samples were prepared in parallel, and 20 chromatographic peaks were randomly selected for continuous injection analysis. The RSD values of the peak area and retention time of the 20 chromatographic peaks were calculated.

For sample stability testing, the same QC sample solution was used for $0,6,12,18$, and $24 \mathrm{~h}$ injection analyses, and 20 chromatographic peaks were randomly selected. RSD values of corresponding peak area and retention times were calculated.

\section{Metabolomics Data Processing}

Mice serum were analyzed by UPLC-Q-TOF/MS. Multivariate statistical analysis and data integration analysis were used to screen terminal metabolites differentially regulated by ZDF in ITP mice. The data analysis process was as follows: first, multivariate data analysis was performed using SIMCA-P +11.5 software. PCA was used to remove outlier samples. Second, supervised PLS-DA was performed, and compounds that had a significant contribution to the classification (VIP > 1) were considered as differentially regulated candidate metabolites. Finally, Independent Samples $t$-test was performed using SPSS 17.0, and $p<0.05$ indicated significance. We identified potential biomarkers based on their $\mathrm{m} / \mathrm{z}$ values, the $\mathrm{HMDB}^{2}$, and the KEGG database and literature $^{3}$. The differential metabolites of ITP mice obtained upon final identification were searched in the drug delivery group, and the effects of $\mathrm{ZDF}$ on these metabolites were recorded.

\section{Statistical Analysis}

Data were expressed as mean \pm standard deviation (SD) and analyzed with SPSS software. Statistically significant values were

${ }^{2} \mathrm{http}: / /$ www.hmdb.ca/

${ }^{3} \mathrm{http}: / /$ www.genome.jp/kegg/ carried out by one-way ANOVA test with post hoc contrasts by Student-Newman-Keuls test. $P<0.05$ indicated statistical significance.

\section{RESULTS}

\section{Identification of Zi Dian Fang's Chemical Constituents and Attribution Analysis}

For comprehensive identification of ZDF chemical constituents, a ZDF test solution was analyzed using UPLC-Q-TOF/MS both in positive and negative ion scanning modes. The corresponding total ion current diagrams are shown in Figures 1A,B. The total ion current diagram of single herbs is shown in Supplementary Figures S1A,B. A total of $52 \mathrm{ZDF}$ constituents were identified, and later subjected to network pharmacological analysis (Supplementary Table S1).

\section{Network Pharmacology Analysis Potential Targets}

Five hundred and twenty ZDF targets were predicted using the Pharm Mapper server ${ }^{4}, 45$ of which were closely related to ITP with a total frequency of 235 (Table 2). Analysis of "chemical composition-target" correlation revealed multiple compounds acting on the same target, and multiple targets affected by the same compound. For instance, the GTPase HRas (Ras) is the target of gallic acid, paeonol, chlorogenic acid, and other chemical components. Astragaloside A simultaneously acts on mitogen-activated protein kinase (MAPK)-activated protein kinase 2, tyrosine-protein kinase ITK/TSK, and protein kinase C-theta, among many other targets. Among the 45 targets closely related to ITP, the Ras target protein appeared with the highest frequency, and it was strongly correlated to platelet proliferation, immunity, and inflammation (Kakunaga et al., 2004; Gunderson et al., 2013; Hauling et al., 2014).

${ }^{4}$ http://lilab.ecust.edu.cn/pharmmapper/ 


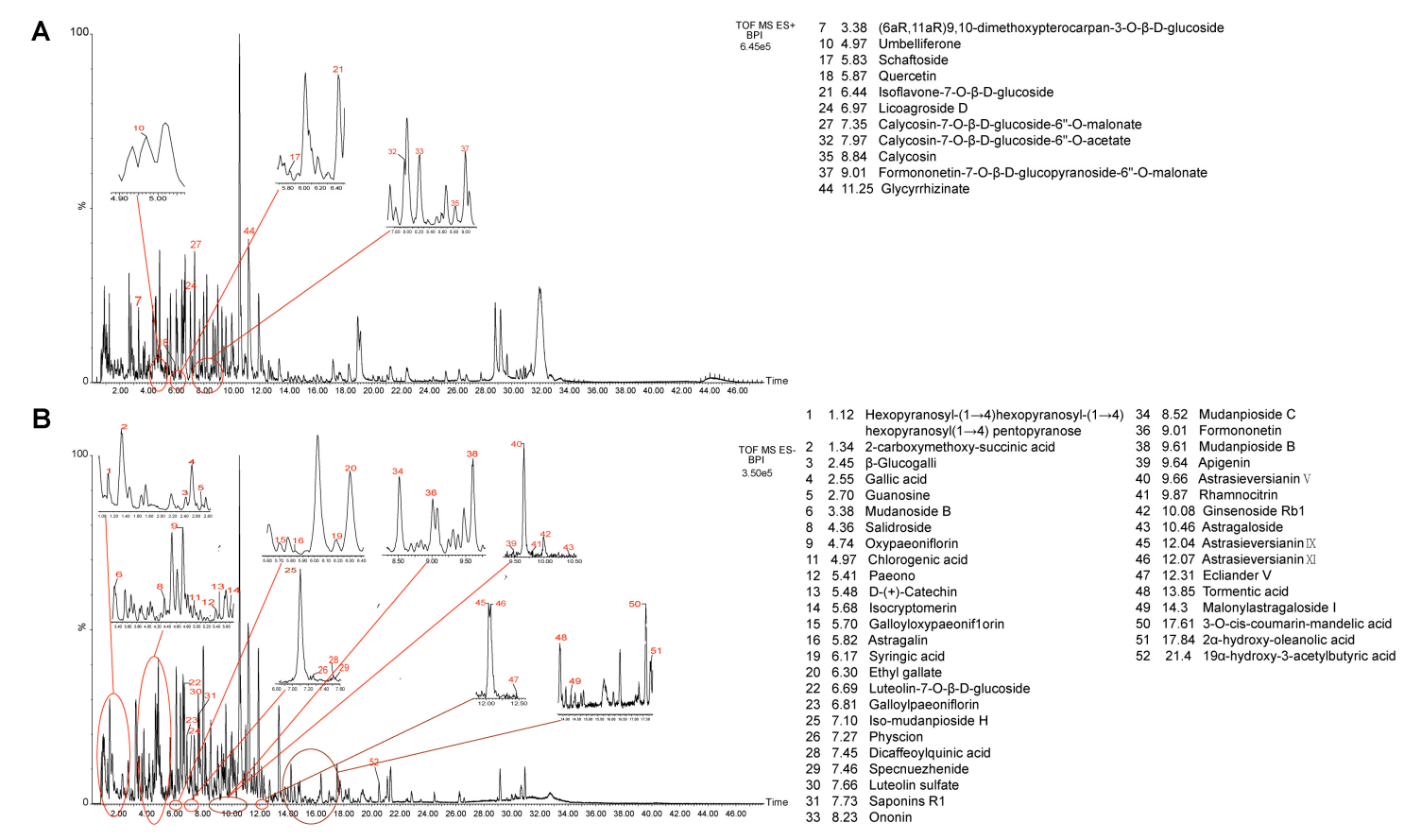

FIGURE 1 | Total ion flow chart of Zi Dian Fang. (A) ESI (+); (B) ESI (-).

\section{Zi Dian Fang Correlative Pathways and Construction of "Chemical Components-Targets-Pathways" Regulatory Network}

Based on the above results, cellular pathways were analyzed by MAS $3.0^{5}$ and the KEGG database ${ }^{6}$, and 113 pathways were identified. Among these, 43 were closely related to ITP. Pathways associated with platelet proliferation included 'hematopoietic cell lineage,' 'complement and coagulation cascade', and 'JAK-STAT signaling pathway', among others (Morin-Poulard et al., 2013; Conway, 2015; Guo et al., 2015). Pathways associated with immunoregulation included 'B cell receptor signaling pathway,' ' $\mathrm{T}$ cell receptor signaling pathway', 'antigen processing and presentation,' 'natural killer cell-mediated cytotoxicity', and 'Tolllike receptor signaling pathway,' among others (Notarangelo, 2014; Zhong et al., 2014; Roche and Furuta, 2015; Goulopoulou et al., 2016; Rajalingam, 2016). Inflammation-related pathways included 'MAPK signaling pathway,' 'VEGF signaling pathway,' and 'AA metabolism' (Ramakrishnan et al., 2014; Schuck et al., 2014; Peng et al., 2016). Cytoscape software was used to establish the "chemical composition-target-pathway" regulatory network of $\mathrm{ZDF}$, which shows the correlation of 52 compounds, 45 target proteins, and 43 ITP-associated pathways (Figure 2A).

There were 27 pathways associated with Ras protein, among which 'B cell receptor signaling pathway', 'T cell receptor signaling pathway', and 'MAPK signaling pathway' were the

${ }^{5} \mathrm{http}: / /$ bioinfo.capitalbio.com/mas3

${ }^{6} \mathrm{http}: / /$ www.genome.jp/kegg/pathway.html most closely related ones. KEGG data visualization made it obvious that the Ras downstream target proteins p38, ERK, and JNK are key connection points between the MAPK signaling pathway and both $\mathrm{T}$ cell and $\mathrm{B}$ cell receptor signaling pathways. Activation of p38, ERK and JNK can affect cell proliferation, immune status, and inflammation. According to the results of network pharmacology, a preliminary prediction of the mechanism by which ZDF treats ITP may be as follows: ZDF inhibits the expression of Ras and prevents phosphorylation of its downstream effectors ERK, JNK, and p38, thus promoting platelet proliferation, immune regulation, and an anti-inflammatory effect. Subsequently, for network pharmacology validation an animal model of ITP was established to detect the key targets in the Ras/MAPKs signaling pathway (Figure 2B), and to observe variation trends of the detection indexes.

\section{In Vivo Verification of Network Pharmacology-Based Virtual Screening Results \\ Effect of Zi Dian Fang on Peripheral Platelet Count, Spleen Index, and Bone Marrow Megakaryocytes Count}

The effect of ZDF on peripheral platelet counts was evaluated in ITP mice on days 7 and 14 after ITP induction by GPAPS injection. Platelet counts in the model group were significantly lower than in the control group. Meanwhile, ITP mice treated with ZDF exhibited increased peripheral platelet counts compared with the model group (Supplementary Table S2). The splenic index in the ITP model group was 
TABLE 2 | Zi Dian Fang (ZDF) major active ingredient related targets.

\begin{tabular}{|c|c|c|c|}
\hline Serial number & Uniport ID & Target protein name & Frequency \\
\hline 1 & P01112 & GTPase HRas & 35 \\
\hline 2 & Q9BZX2 & Uridine-cytidine kinase 2 & 26 \\
\hline 3 & P09211 & Glutathione S-transferase P & 17 \\
\hline 4 & P27707 & Deoxycytidine kinase & 14 \\
\hline 5 & P18031 & Tyrosine-protein phosphatase non-receptor type 1 & 10 \\
\hline 6 & P29218 & Inositol monophosphatase 1 & 11 \\
\hline 7 & P24941 & Cell division protein kinase 2 & 9 \\
\hline 8 & Q08499 & cAMP-specific 3,5-cyclic phosphodiesterase 4D & 9 \\
\hline 9 & P49841 & Glycogen synthase kinase- 3 beta & 8 \\
\hline 10 & P08473 & Neprilysin & 9 \\
\hline 11 & P12931 & Proto-oncogene tyrosine-protein kinase Src & 7 \\
\hline 12 & Q02750 & Dual specificity mitogen-activated protein kinase kinase 1 & 6 \\
\hline 13 & P08246 & Neutrophil elastase & 5 \\
\hline 14 & Q00534 & Cell division protein kinase 6 & 5 \\
\hline 15 & 015530 & 3-phosphoinositide-dependent protein kinase 1 & 4 \\
\hline 16 & Q16539 & Mitogen-activated protein kinase 14 & 4 \\
\hline 17 & P35558 & Phosphoenolpyruvate carboxykinase, cytosolic [GTP] & 4 \\
\hline 18 & P15121 & Aldose reductase & 5 \\
\hline 19 & Q06187 & Tyrosine-protein kinase BTK & 3 \\
\hline 20 & P02679 & Fibrinogen gamma chain & 3 \\
\hline 21 & P11712 & Cytochrome P450 2C9 & 3 \\
\hline 22 & P00492 & Hypoxanthine-guanine phosphoribosyltransferase & 3 \\
\hline 23 & P36873 & Serine/threonine-protein phosphatase PP1-gamma catalytic subunit & 3 \\
\hline 24 & P49137 & MAP kinase-activated protein kinase 2 & 3 \\
\hline 25 & Q08881 & Tyrosine-protein kinase ITK/TSK & 3 \\
\hline 26 & P20248 & Cyclin-A2 & 2 \\
\hline 27 & P11362 & Basic fibroblast growth factor receptor 1 & 2 \\
\hline 28 & Q03518 & Antigen peptide transporter 1 & 2 \\
\hline 29 & P00918 & Carbonic anhydrase 2 & 2 \\
\hline 30 & P53779 & Mitogen-activated protein kinase 10 & 2 \\
\hline 31 & P11309 & Serine/threonine-protein kinase pim-1 & 2 \\
\hline 32 & Q04759 & Protein kinase $\mathrm{C}$ theta type & 1 \\
\hline 33 & P14555 & Phospholipase A2, membrane associated & 1 \\
\hline 34 & P08709 & Coagulation factor VII & 1 \\
\hline 35 & P03951 & Coagulation factor $\mathrm{XI}$ & 1 \\
\hline 36 & P35968 & Vascular endothelial growth factor receptor 2 & 1 \\
\hline 37 & P31749 & RAC-alpha serine/threonine-protein kinase & 1 \\
\hline 38 & P42574 & Caspase-3 & 1 \\
\hline 39 & 060760 & Glutathione-requiring prostaglandin D synthase & 1 \\
\hline 40 & P42330 & Aldo-keto reductase family 1 member C3 & 1 \\
\hline 41 & P15056 & Serine/threonine-protein kinase B-raf & 1 \\
\hline 42 & Q13231 & Chitotriosidase-1 & 1 \\
\hline 43 & P28161 & Glutathione S-transferase Mu 2 & 1 \\
\hline 44 & Q00688 & Peptidyl-prolyl cis-trans isomerase FKBP3 & 1 \\
\hline 45 & P08581 & Hepatocyte growth factor receptor & 1 \\
\hline
\end{tabular}

significantly higher than in the control group, and was reduced by ZDF treatment (Supplementary Table S3), indicating effective reduction of ITP-induced splenomegaly in ITP mice. The total number of bone marrow megakaryocytes was increased in the ITP model group compared with control mice. Specifically, the number of thrombogenic megakaryocytes was decreased and the number of granular megakaryocytes was increased. The total number and classification of bone marrow megakaryocytes in the different groups are listed in Supplementary Table S4. These results suggest active bone marrow megakaryocyte proliferation in ITP mice, accompanied by anomalous maturation reflected by increased numbers of granular megakaryocytes and decreased numbers of thrombogenic megakaryocytes. ZDF administration 

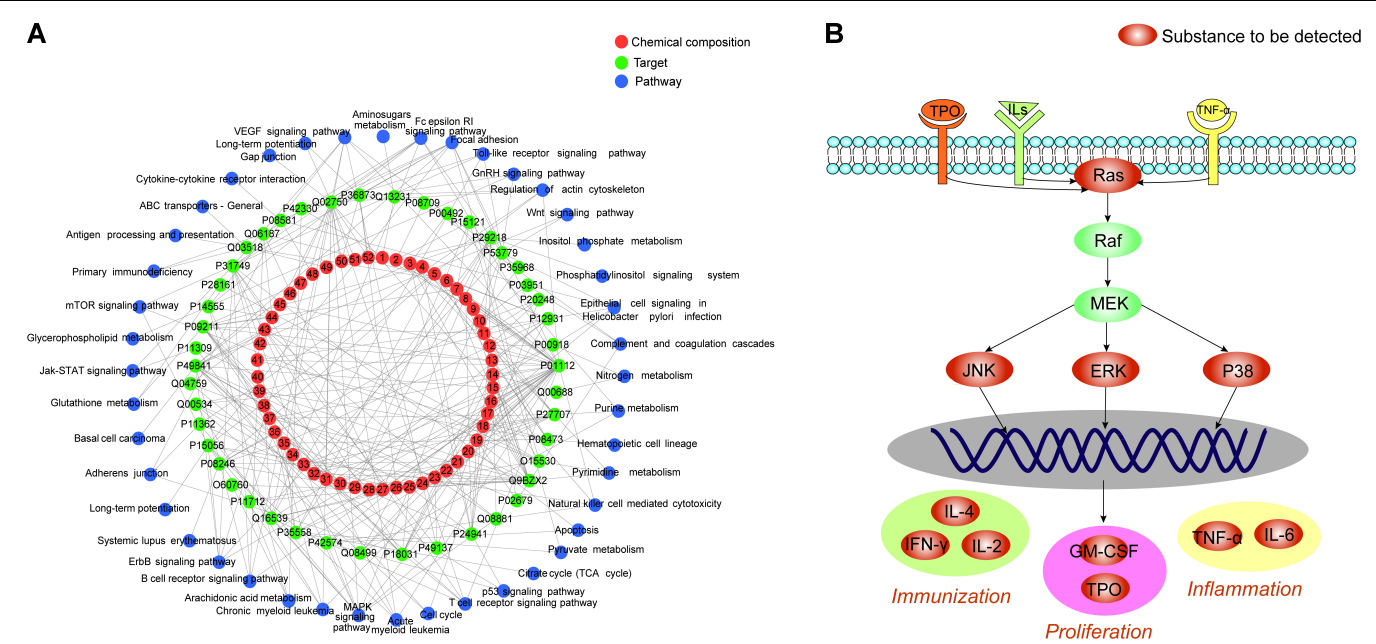

FIGURE 2 | (A) Zi Dian Fang's "chemical composition-target-pathway" regulatory network. Numbers 1-52 circled in red represent chemical composition, and correspond to data on Figure 1. (B) Identified Ras/MAPKs signaling pathway proteins.

largely normalized these changes, indicating a therapeutic effect.

While prednisone's beneficial effects on platelet and megakaryocyte counts were similar to those of ZDF, analysis of the spleen index showed that prednisone acetate significantly decreased spleen index compared with the control group, but the spleen index in the treatment group did not decrease significantly compared with the control group. Thus, ZDF may have the advantage of alleviating the adverse effects of immunosuppression associated with prednisone use.

\section{Western Blot and Enzyme-Linked Immunosorbent Assay Analysis}

Ras signaling pathway plays a critical role in the $\mathrm{T}$ and $\mathrm{B}$ cell receptor and MAPK signaling pathways. The Ras/MAPK pathway effectors ERK1/ERK2, JNK, and p38 are important members of the MAPK family (Gao et al., 2017). Under normal physiological conditions, these proteins are dephosphorylated and signal transduction does not occur. When cells are stimulated (i.e., during inflammation, immune challenge, etc.), Ras activation leads to downstream ERK, JNK, and p38 phosphorylation and activation of the Ras/MAPKs signaling pathway, which promotes the expression of cell factors associated with immunoregulation (IL-2, IFN- $\gamma$ ), platelet proliferation (TPO, GM-CSF), and inflammation (TNF- $\alpha$, IL-6) (Kitanaka et al., 2017).

To correlate ZDF network pharmacology predictions with in vivo ZDF effects, splenic levels of Ras, ERK, JNK, and p38, and their corresponding phosphorylated forms were verified by western blot. Compared with the control group, Ras, p-JNK/JNK, p-ERK/ERK, and p-p38/p38 levels were significantly increased in the model group, while these protein expression changes were attenuated by treatment with ZDF (Figures 3A-E). Next, ELISA assays were carried out in serum samples to analyze molecular markers associated with proliferation, i.e., TPO and GM-CSF, inflammation, i.e., TNF- $\alpha$ and IL-6, and immune response, i.e.,
IL-2, IFN- $\gamma$ and IL-4. As shown in Figure 3B, IL-2, IFN$\gamma$, TPO, GM-CSF, TNF- $\alpha$, and IL- 6 levels were significantly increased, while IL- 4 production was significantly decreased, in the model group compared with the control group, while all these substances were differentially regulated in the ZDF treatment group.

\section{Metabolomics Analysis}

Method precision and sample stability tests were first carried out using serum QC samples in positive ion mode. The base peak chromatogram (BPC) is shown in Supplementary Figure S2. Results showed that peak area were less than $15 \%$ and retention time RSD values were less than $5 \%$, indicating that instrument precision, method precision and sample stability were reliable. Specific information is shown in Supplementary Table S5.

Metabolomics studies were performed using multivariate statistical analysis. First, PCA was used for unsupervised data analysis (Figure 4A) but showed no clear distinction between the control, model, and treatment groups. Therefore, we carried out supervised data analysis using PLS-DA (Figure 4B), which showed good distinction between the three groups. This analysis indicated that endogenous metabolite levels in the model group were significantly different compared with the control group. After ZDF administration, metabolite levels differed in turn significantly from the model group, approaching the levels observed in the control group. Based on the PLS-DA analysis, variables with VIP $>1$ between the control and model groups were selected as potential biomarkers associated with ITP, and their significance assessed through Student's $t$-test.

Twelve differentially regulated metabolites were identified with basis on their variation in the ZDF treatment groups and available literature reports. Among these, leucine, arginine, palmitoleic acid, stearic acid, and 5-aminoimidazole ribonucleotides, and phenylpyruvic acid levels were significantly 

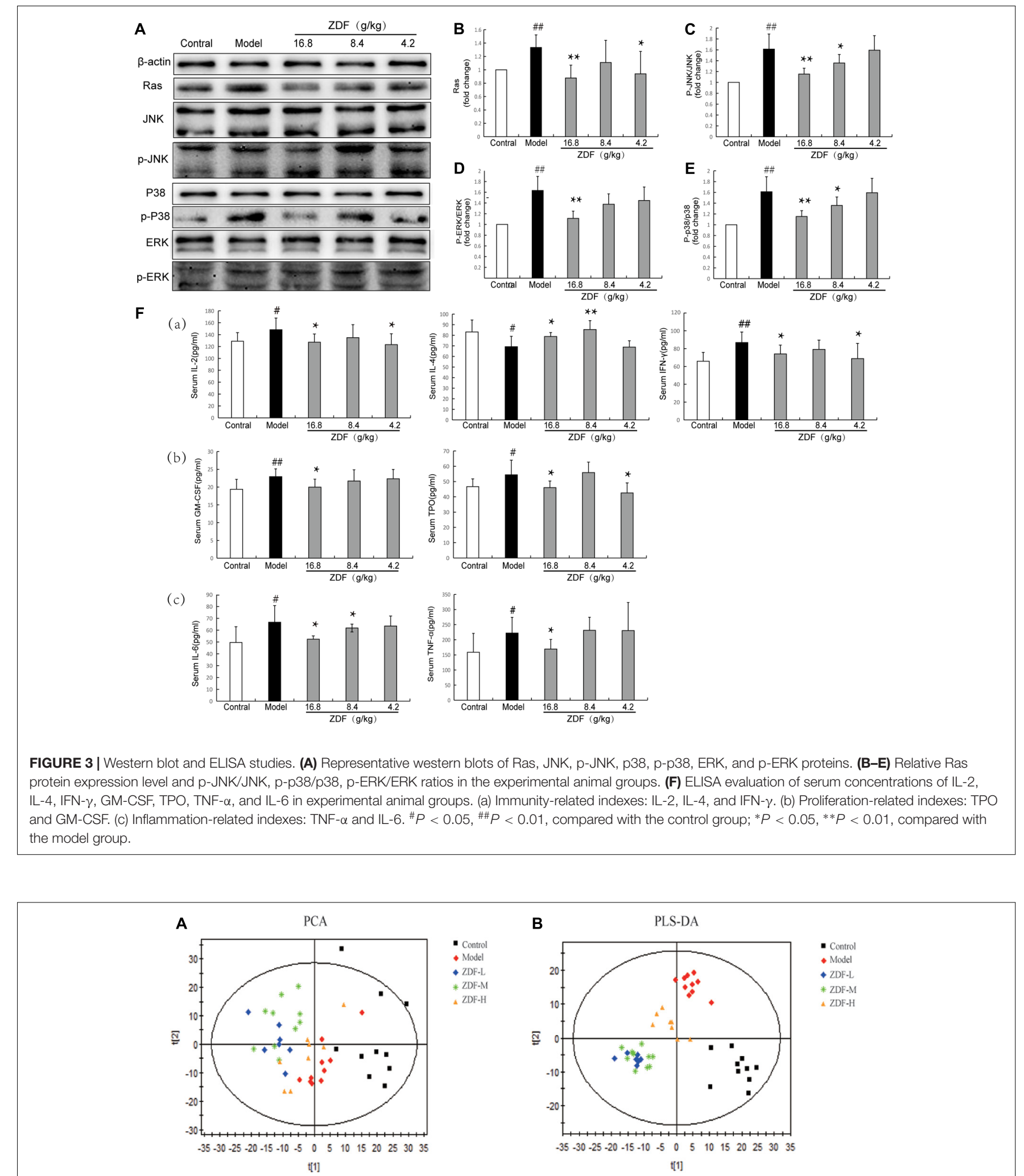

FIGURE 4 | Multivariate analysis results. (A) PCA score plots of the model group compared with the control group, and the ZDF-L, ZDF-M, and ZDF-H treatment groups compared with the model group. (B) PLS-DA score plots of the model group compared with the control group, and the ZDF-L, ZDF-M, and ZDF-H treatment groups compared with the model group. ZDF-L: ZDF low dose group; ZDF-M: ZDF middle dose group; ZDF-H: ZDF high dose group. 
decreased, while S1P, 9-cis-retinoic acid, EPA, and $\Delta^{12}$-PGJ2 levels were significantly increased in the model group compared with the control group. All these metabolites were differentially regulated in the ZDF treatment group. Detailed information is shown in Table 3.

\section{Network Pharmacology and Metabolomics Integration Analysis}

To integrate network pharmacology and metabolomics data, main target proteins and differentially regulated metabolites within the Ras/MAPKs signaling pathway were respectively screened by these methods. Then, the ID number of target proteins and differential metabolites were searched on the KEGG database to find shared metabolic pathways, and results combined with the literature to find reported relationships. Overall analysis results preliminarily confirmed that ZDF therapeutic actions on ITP are based on inhibition of the Ras/MAPKs signaling pathway and attenuation of immune and inflammatory responses and promotion of platelets proliferation. The correlation between target proteins and differential metabolites affected by ZDF is illustrated in Figure 5.

\section{DISCUSSION}

\section{Platelet Homeostasis and Proliferation}

Through different techniques, we demonstrated that the level of Ras and p-ERK/ERK in spleen of the model group was significantly higher than that in the control group (Figures 3B,D), serum TPO, GM-CSF, S1P, EPA, and $\Delta^{12}$-PGJ2 levels were significantly increased and arginine was significantly decreased in the model group compared with the control group, while all these substances were differentially regulated in the ZDF treatment group.

Ras protein is a key upstream protein in the Ras/MAPKs signaling pathway and it is an important component in regulating cell growth, proliferation and differentiation. ERK is downstream protein of the Ras/MAPKs signaling pathway. $\mathrm{S} 1 \mathrm{P}$ is a biologically active sphingolipid that acts as an extracellular ligand for S1P membrane receptors (S1P1-5) and affects cell survival, proliferation, and differentiation through distinct signal transduction pathways (Mendelson et al., 2014). TPO is a specific growth factor that stimulates proliferation and differentiation of megakaryocytes and hence regulates platelet production (Mazza et al., 2016). When the number of peripheral platelets is high, TPO uptake increases, serum TPO decreases, and proliferation, differentiation, and maturation of megakaryocytes is inhibited, resulting in decreased platelet production (Hitchcock and Kaushansky, 2014). In the course of ITP, peripheral platelet levels decrease and serum TPO increases. TPO binds to its receptor and activates the Ras/ERK signaling pathway, leading to S1P generation (Garcia et al., 2001). S1P binds to its G protein-coupled receptor(s) and further activates the Ras/ERK signaling pathway, leading to an increase in GM-CSF, which in turn stimulates differentiation of myeloid progenitor cells (Na et al., 2016; Kraakman et al.,
2017). Elevated levels of TPO and GM-CSF also promote macrophage maturation. Activated macrophages are classified into two types: classically activated macrophages (M1) and alternatively activated macrophages (M2) (Genin et al., 2015). M1 cells contain NOS, which catalyzes the oxidation of the essential amino acid arginine to NO, which inhibits platelet aggregation (Mazzanti et al., 2011; Roy et al., 2015). The same effect is elicited by EPA, one of the most abundant and essential unsaturated fatty acids (Ohnishi and Saito, 2013). EPA-mediated inhibition of platelet aggregation is related to the metabolism of AA (Roy et al., 2016). AA is metabolized by COX to form two substances with opposing actions: PGI2, a $\Delta^{12}$-PGJ2 precursor that inhibits platelet aggregation, and TXA2, which is vasoconstrictor and promotes platelet aggregation (Kashiwagi et al., 2015). Studies have shown that EPA can inhibit the formation of TXA2, thereby inhibiting platelet aggregation (Schmitz et al., 2000). However, while NO and EPA both inhibit platelet aggregation, a simultaneous rise in these two substances can lead to coagulation dysfunction and aggravate symptoms in ITP patients.

Our data suggests that the mechanism by which ZDF promotes platelet proliferation and improves coagulation in patients with ITP may be as follows: on the one hand, ZDF reduces serum TPO concentration and inhibits Ras/ERK signaling pathway activation, so that downstream GM-CSF and S1P expression decreases, resulting in reduced platelet activation and increased platelet proliferation. On the other hand, ZDF-mediated Ras/ERK signaling inhibition reduces the number of M1 cells, leading to lower NOS activity, reduced arginine consumption and NO production, and reduced EPA and $\Delta^{12}$-PGJ2 synthesis through AA metabolism. All these changes counteract defective platelet aggregation and improve coagulation, thus reducing ITP symptoms.

\section{Immunoregulation}

Present results showed that the concentration of immunerelated cytokines IL- 2 and IFN- $\gamma$ in the serum of ITP mice was significantly higher than in the control group, while the content of IL-4 was instead decreased. In addition, leucine, arginine, and phenylpyruvic acid levels were decreased (Figure 3F), while S1P, 9-cis-retinoic acid, and oleic acid amide levels were increased, in the model group. Levels of all these substances were differentially regulated in the ZDF treatment group.

Immune thrombocytopenic purpura etiology is closely related to abnormal immune function, particularly involving Th1/Th2 cell dysfunction (Yao et al., 2014). IL-2, IFN- $\gamma$, and IL-4 synthesis is regulated by the Ras/MAPKs signaling pathway in Th1 and Th2 cells. In general terms, IL- 2 and IFN- $\gamma$ are produced by activated Th1 cells and mediate cellular immune responses, while IL-4 is produced by activated Th2 cells and mediates humoral immune responses (Hall, 2015; Almeida et al., 2017). Leucine is the most common amino acid found in proteins, and can indirectly promote the release of IL-4 (Sahoo et al., 2015). Consistent with Th1/Th2 dysfunction, leucine deficiency leads to decreased IL-4 production, weakening of the humoral immune response, and stimulation of the cellular immune response (Bassit 
TABLE 3 | Identified potential biomarkers for the discrimination between model group and control group in serum samples.

\begin{tabular}{|c|c|c|c|c|c|c|c|c|c|}
\hline \multirow[t]{2}{*}{ No. } & \multirow[t]{2}{*}{ Rt (min) } & \multirow[t]{2}{*}{$(\mathrm{m} / \mathrm{z})$} & \multirow[t]{2}{*}{ Adduct } & \multirow[t]{2}{*}{ Metabolites } & \multirow[t]{2}{*}{ Formula } & \multicolumn{4}{|c|}{ Trend } \\
\hline & & & & & & $\begin{array}{l}\text { Model Group vs. } \\
\text { control group }\end{array}$ & $\begin{array}{l}\text { ZDF-L vs. } \\
\text { Model Group }\end{array}$ & $\begin{array}{l}\text { ZDF-M vs. } \\
\text { Model Group }\end{array}$ & $\begin{array}{l}\text { ZDF-Hvs. } \\
\text { Model Group }\end{array}$ \\
\hline 1 & 1.20 & 296.065 & $\mathrm{M}+\mathrm{H}$ & 5-aminoimidazole ribonucleotide & $\mathrm{C}_{8} \mathrm{H}_{14} \mathrm{~N}_{3} \mathrm{O}_{7} \mathrm{P}$ & $\downarrow^{\# \#}$ & $\downarrow$ & $\downarrow$ & $\uparrow^{*}$ \\
\hline 2 & 1.24 & 198.0859 & $\mathrm{M}+\mathrm{Na}$ & Argininic acid & $\mathrm{C}_{6} \mathrm{H}_{13} \mathrm{~N}_{3} \mathrm{O}_{3}$ & $\downarrow^{\# \#}$ & $\uparrow$ & $\uparrow$ & $\uparrow$ \\
\hline 3 & 1.56 & 165.0573 & $\mathrm{M}+\mathrm{H}$ & Phenylpyruvic acid & $\mathrm{C}_{9} \mathrm{H}_{8} \mathrm{O}_{3}$ & $\downarrow^{\# \#}$ & $\uparrow$ & $\uparrow^{* *}$ & $\uparrow^{*}$ \\
\hline 4 & 1.56 & 132.101 & $\mathrm{M}+\mathrm{H}$ & $( \pm)$-leucine & $\mathrm{C}_{6} \mathrm{H}_{13} \mathrm{NO}_{2}$ & $\downarrow^{\# \#}$ & $\uparrow^{*}$ & $\uparrow^{* *}$ & $\uparrow * *$ \\
\hline 5 & 4.54 & 357.2039 & $\mathrm{M}+\mathrm{Na}$ & Delta-12-prostaglandin j2 & $\mathrm{C}_{20} \mathrm{H}_{30} \mathrm{O}_{4}$ & $\uparrow^{\#}$ & $\uparrow$ & $\uparrow$ & $\downarrow^{*}$ \\
\hline 6 & 5.44 & 402.2389 & $\mathrm{M}+\mathrm{Na}$ & Sphingosine 1-phosphate & $\mathrm{C}_{18} \mathrm{H}_{38} \mathrm{NO}_{5} \mathrm{P}$ & $\uparrow^{\#}$ & $\downarrow$ & $\downarrow$ & $\downarrow^{*}$ \\
\hline 7 & 6.05 & 301.2173 & $\mathrm{M}+\mathrm{H}$ & 9-cis-Retinoic acid & $\mathrm{C}_{20} \mathrm{H}_{28} \mathrm{O}_{2}$ & $\uparrow \uparrow^{\# \#}$ & $\uparrow^{*}$ & $\uparrow$ & $\uparrow$ \\
\hline 8 & 6.39 & 263.2389 & $\mathrm{M}+\mathrm{Na}$ & Palmitaldehyde & $\mathrm{C}_{16} \mathrm{H}_{32} \mathrm{O}$ & $\downarrow^{\# \#}$ & $\uparrow$ & 个* & $\uparrow *$ \\
\hline 9 & 6.88 & 282.2792 & $\mathrm{M}+\mathrm{H}$ & Oleamide & $\mathrm{C}_{18} \mathrm{H}_{35} \mathrm{NO}$ & $\uparrow^{\#}$ & $\downarrow^{*}$ & $\downarrow$ & $\downarrow$ \\
\hline 10 & 8.68 & 277.2144 & $\mathrm{M}+\mathrm{Na}$ & Palmitoleic acid & $\mathrm{C}_{16} \mathrm{H}_{30} \mathrm{O}_{2}$ & $\downarrow^{\# \#}$ & $\uparrow$ & $\uparrow^{*}$ & $\uparrow * *$ \\
\hline 11 & 8.68 & 277.2144 & $\mathrm{M}+\mathrm{H}$ & Stearidonic acid & $\mathrm{C}_{18} \mathrm{H}_{28} \mathrm{O}_{2}$ & $\downarrow^{\# \#}$ & $\uparrow^{* *}$ & $\uparrow^{*}$ & $\uparrow^{* *}$ \\
\hline 12 & 9.18 & 303.2316 & $\mathrm{M}+\mathrm{H}$ & Eicosapentaenoic acid & $\mathrm{C}_{20} \mathrm{H}_{30} \mathrm{O}_{2}$ & $\downarrow^{\#}$ & $\uparrow$ & $\downarrow$ & $\uparrow^{*}$ \\
\hline
\end{tabular}

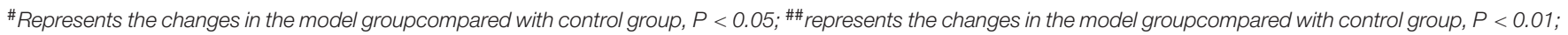

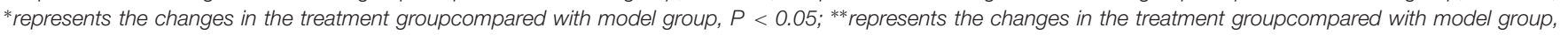

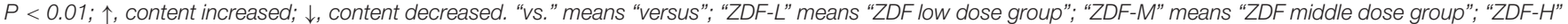
means "ZDF high dose group".

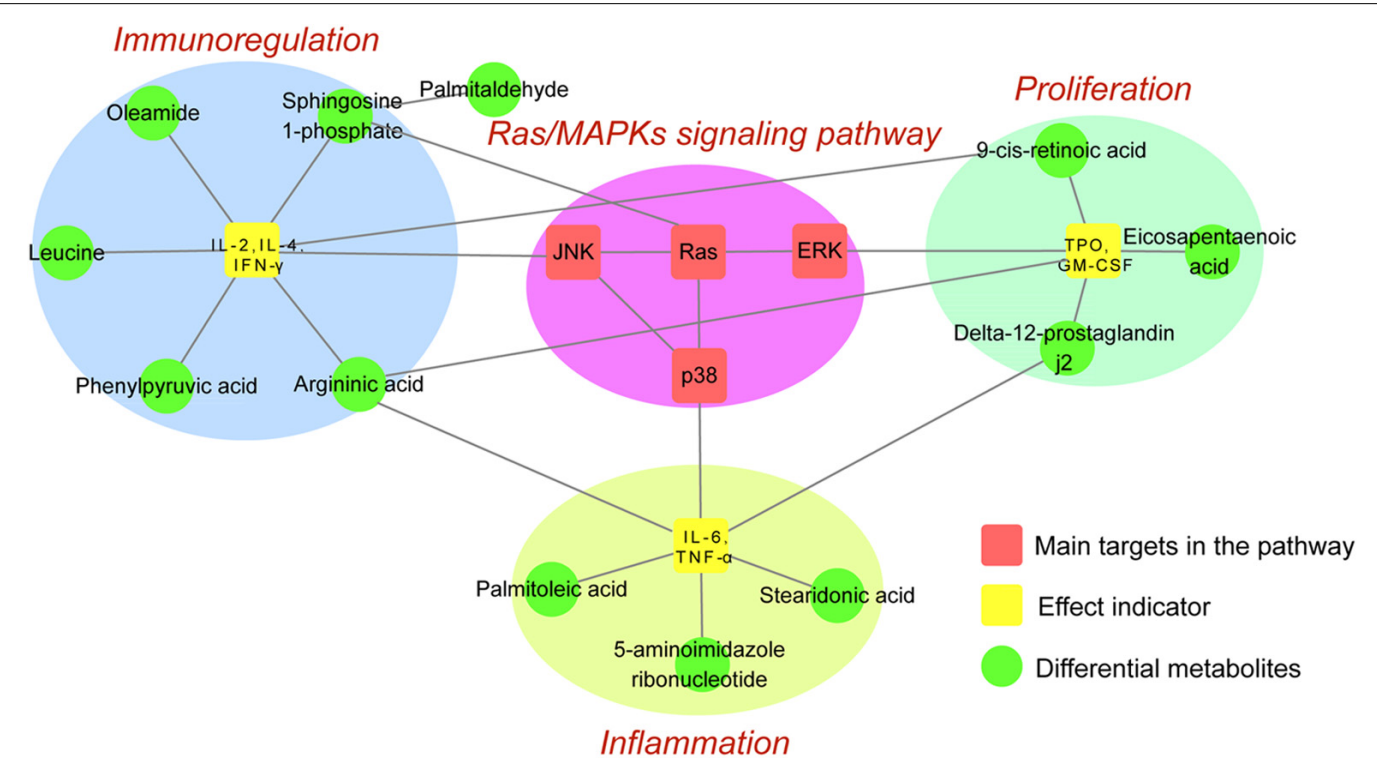

FIGURE 5 | Network map of the Ras/MAPKs signaling pathway and differential metabolites.

et al., 2002). S1P is widely found in blood, lymph, erythrocytes, neutrophils, platelets, and other cells. S1P4 receptor balances Th1/Th2 functions by inhibiting IL-4 secretion by Th2 cells and stimulating IL-10 production by Th1 cells (Wang et al., 2005; Walsh et al., 2014). Differential metabolism of arginine can also enhance the activity of Th cells by stimulating NOSmediated NO production, which promotes apoptosis and inhibits proliferation and activation of T cells (Bonham et al., 2010; Raber et al., 2014). Reduced $\mathrm{T}$ cell proliferation and activation can also be elicited by oleamide, a long-chain fatty acid amide that increases intracellular $\mathrm{Ca}^{2+}$ concentration in T cells (MurilloRodríguez et al., 2001). KEGG pathway database analysis indicated that increases in IL-2 and IFN- $\gamma$ are associated with increased 9-cis-retinoic acid content, leading to an imbalance in Th1/Th2 activities. Meanwhile, the metabolism of phenyl pyruvate, another metabolite found to be decreased in our ITP model, is closely related to that of tryptophan, which has been linked to immune function regulation (Capuron et al., 2014).

Our analyses suggest that ZDF balances Th1/Th2 activities in patients with ITP by inhibiting the release of the Th1-type cytokines IL-2 and IFN- $\gamma$ (thus reducing 9-cis-retinoic acid levels), by promoting IL-4 release, and by inhibiting IL-10 release by upregulation of arginine and downregulation of S1P and oleic 


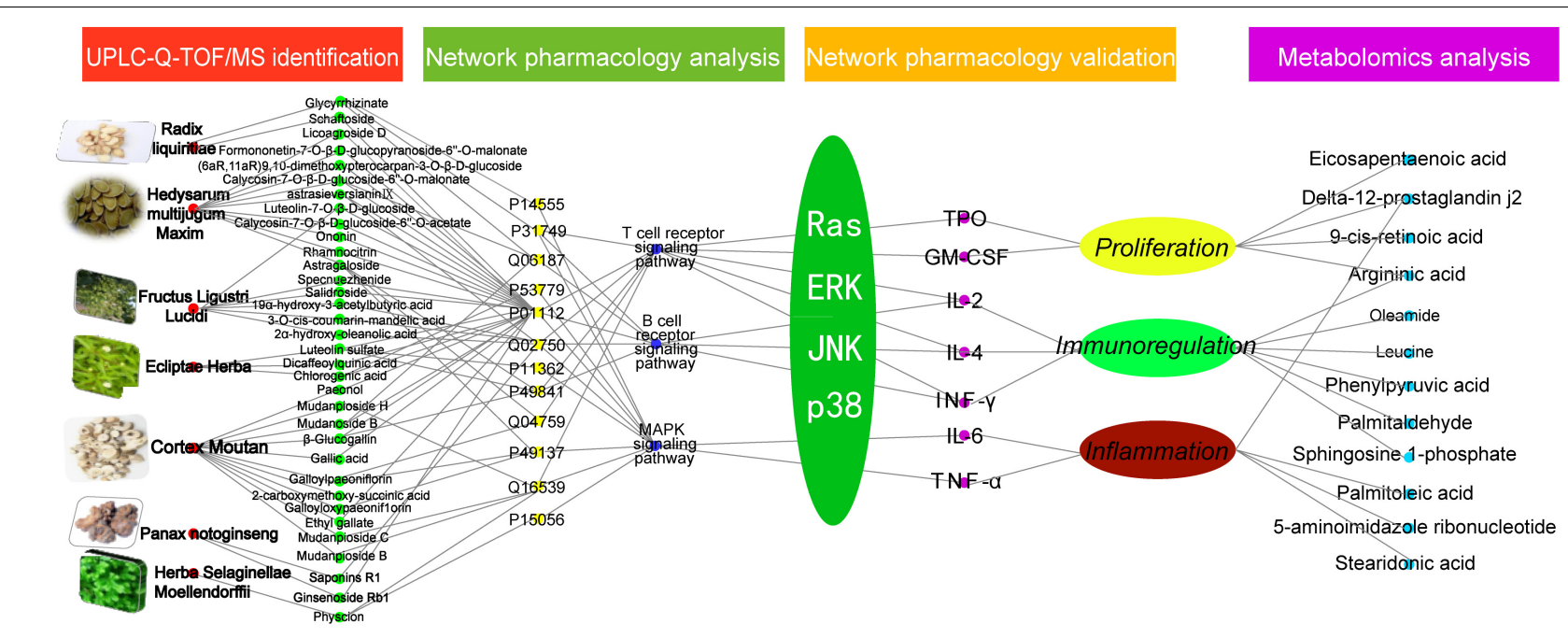

FIGURE 6 | Network pharmacology prediction and metabolomics analysis.

acid amide. On the other hand, ZDF may improve the immune response by upregulating phenylpyruvic acid synthesis.

\section{Anti-inflammatory Effects}

Ras/MAPKs signaling activation is closely linked to inflammatory stimuli (Kim et al., 2015; Hou et al., 2016), leading to production of cytokines such as IL-6 and TNF- $\alpha$ (Li et al., 2016). IL-6 is a lymphokine produced by activated $\mathrm{T}$ cells and fibroblasts, which regulates the inflammatory response (Chaturvedi et al., 2015). Starkie et al. indicate that skeletal muscle markedly releases IL-6, which could exert anti-inflammatory effects through the downregulation of TNF- $\alpha$ activity during "non-damaging" exercise (Starkie et al., 2003). When ITP occurs, the body is in an inflammatory state (Dal et al., 2015). In the course of inflammation, naive $\mathrm{T}$ cells differentiate into Th17 cells in the presence of TGF- $\beta 1$ and IL-6. IL- 6 inhibits the development of Treg cells and promotes the development of inflammatory responses (Bettelli et al., 2006). In our study, serum IL-6 and TNF- $\alpha$ levels were significantly increased in ITP mice (Figure 3F), which is consistent with the literature reports (Talaat et al., 2014). We consider that IL-6 may play a proinflammatory role in the ITP model. TNF- $\alpha$ has critical functions in the inflammatory microenvironment, and its cytotoxic effect is related to activation of phospholipase A2, which releases AA from membrane phospholipids (Ahn et al., 2015). AA, which may also be generated from stearic acid desaturation to oleic acid (Harvey et al., 2010), can lead to oxidative stress and initiation of chronic inflammatory responses by its metabolism through COX and lipoxygenase (LOX). Since COX-2 is the ratelimiting enzyme in the synthesis of prostaglandins from AA in macrophages, leukocytes, and fibroblasts, we speculate that the observed downregulation of stearic acid and upregulation of $\Delta^{12}$-PGJ2 expression in ITP mice may be related to COX-2 overexpression. $\Delta^{12}$-PGJ2 is a potent inflammatory mediator that promotes the release of IL- 6 and TNF- $\alpha$ (Heinemann et al., 2003). Inflammation also stimulates iNOS in immune and endothelial cells, which generate large amounts of NO that positively affect further expression of iNOS and exacerbate inflammation (Guzik et al., 2003). Palmitoleic acid (9-hexadecenoic acid) belongs to the same unsaturated fatty acid family of stearic acid. Both fatty acids were down regulated in model group mice, suggesting that impaired lipid metabolism may contribute to the inflammatory response in ITP mice (van Diepen et al., 2013).

Based on the present findings, we suggest that $\mathrm{ZDF}$ reduces inflammation in ITP mice by lowering the production of proinflammatory cytokines TNF- $\alpha$ and IL-6 through inhibition of p38, ERK, and JNK activation, downregulation of $\Delta^{12}$-PGJ2, and upregulation of palmitoleic acid, stearic acid, and arginine levels.

\section{CONCLUSION}

In conclusion, using UPLC, mass spectroscopy-based metabolomics, western blot, ELISA, and network pharmacology the present study analyzed the composition of the TCM prescription ZDF, and the changes it induced on serum metabolites and cellular signaling pathways in an animal model of ITP. Following identification of 52 chemical constituents in the ZDF formulation by UPLC-Q-TOF/MS, network pharmacology predicted significant pharmacological actions centered on three main pathophysiological aspects: platelet proliferation, immunoregulation, and inflammation. After validation of predicted main targets and pathways through animal experiments, we proposed a plausible mechanism for the multi-target effects of ZDF on ITP (Figure 6). According to the proposed model, ZDF inhibits Ras expression and phosphorylation of its downstream effectors, ERK, JNK, and p38, attenuating the release of cell factors associated with proliferation (TPO, GM-CSF), inflammation (TNF- $\alpha$, IL6), and cell-mediated immunity (IL-2, IFN- $\gamma$ ). Meanwhile, stimulation of IL-4 production promotes platelet proliferation, balances Th1/Th2 immune responses, and reduces inflammatory 
reactions. This study provides data support for the in-depth study of the mechanisms of ZDF in the treatment of ITP and lays a foundation for the clinical application of ZDF. We believe that ZDF provides an effective strategy and may be a useful alternative to hormone-based therapies for the treatment of ITP.

\section{AUTHOR CONTRIBUTIONS}

YuL and YWa helped with study design, data interpretation and writing of manuscripts. WL and HL help statistical analysis and editing manuscripts. YaL and HL help to experiment, edit manuscripts, and interpret data. YWu, WD, and GB help writing manuscripts. YZ designed, performed and funded all

\section{REFERENCES}

Ahn, M. Y., Kim, S. J., Kim, N., Hwang, J. S., and Yun, E. Y. (2015). Immune modulation of glycosaminoglycan derived from $P$. lewisi in TNF- $\alpha$ stimulated cells. Arch. Pharm. Res. 38, 1983-1991. doi: 10.1007/s12272-0150616-5

Almeida, R. D. S., Ramos, A. M. L., Luna, C. F., Pedrosa, F., Donadi, E. A., and Lucena-Silva, N. (2017). Cytokines and soluble HLA-G levels in bone marrow stroma and their association with the survival rate of patients exhibiting childhood T-cell acute lymphoblastic leukemia. Cytokine 102, 94-101. doi: 10.1016/j.cyto.2017.07.014

Arnett, M. G., Muglia, L. M., Laryea, G., and Muglia, L. J. (2016). Genetic approaches to hypothalamic-pituitary-adrenal axis regulation. Neuropsychopharmacology 41, 245-260. doi: 10.1038/npp.2015.215

Bassit, R. A., Sawada, L. A., Bacurau, R. F., Navarro, F., Martins, E. Jr., Santos, R. V., et al. (2002). Branched-chain amino acid supplementation and the immune response of long-distance athletes. Nutrition 18, 376-379. doi: 10.1016/S08999007(02)00753-0

Bettelli, E., Carrier, Y., Gao, W., Korn, T., Strom, T. B., Oukka, M., et al. (2006). Reciprocal developmental pathways for the generation of pathogenic effector TH17 and regulatory T cells. Nature 441, 235-238. doi: 10.1038/nature04753

Bonham, K., Hemmers, S., Lim, Y. H., Hill, D. M., Finn, M. G., and Mowen, K. A. (2010). Effects of a novel arginine methyltransferase inhibitor on T-helper cell cytokine production. FEBS J. 277, 2096-2108. doi: 10.1111/j.1742-4658.2010. 07623.x

Capuron, L., Geisler, S., Kurz, K., Leblhuber, F., Sperner-Unterweger, B., and Fuchs, D. (2014). Activated immune system and inflammation in healthy ageing: relevance for tryptophan and neopterin metabolism. Curr. Pharm. Des. 20, 6048-6057. doi: 10.2174/1381612820666140317110217

Chaturvedi, S., Siegel, D., Wagner, C. L., Park, J., Velde, H., Vermeulen, J., et al. (2015). Development and validation of panoptic Meso scale discovery assay to quantify total systemic interleukin-6. Br. J. Clin. Pharmacol. 80, 687-697. doi: 10.1111/bcp.12652

Chen, H. Y., Lin, Y. H., Hu, S., Yang, S. H., Chen, J. L., and Chen, Y. C. (2015). Identifying chinese herbal medicine network for eczema: implications from a nationwide prescription database. Evid. Based Complement. Alternat. Med. 2015:347164. doi: 10.1155/2015/347164

Conway, E. M. (2015). Reincarnation of ancient links between coagulation and complement. J. Thromb. Haemost. 13(Suppl. 1), S121-S132. doi: 10.1111/jth. 12950

Dal, M. S., Karakus, A., Aydin, B. B., Ekmen, M. O., Ulas, T., and Ayyildiz, O. (2015). Serum uric acid and inflammation in patients with immune thrombocytopenic purpura: preliminary results. Eur. Rev. Med. Pharmacol. Sci. 19, 4385-4389.

Falvo, J. V., Jasenosky, L. D., Kruidenier, L., and Goldfeld, A. E. (2013). Epigenetic control of cytokine gene expression: regulation of the TNF/LT locus and T helper cell differentiation. Adv. Immunol. 118, 37-128. doi: 10.1016/B978-012-407708-9.00002-9 experiments, analyzed and interpreted data and wrote the manuscript.

\section{FUNDING}

This project was supported by Program for Changjiang Scholars and Innovative Research Team in University (IRT_14R41).

\section{SUPPLEMENTARY MATERIAL}

The Supplementary Material for this article can be found online at: https://www.frontiersin.org/articles/10.3389/fphar. 2018.00597/full\#supplementary-material

Gao, F., Li, F., Miao, Y., Xu, L. J., Zhao, Y., Li, Q., et al. (2017). Involvement of the MEK-ERK/p38-CREB/c-fos signaling pathway in Kir channel inhibitioninduced rat retinal Müller cell gliosis. Sci. Rep. 7:1480. doi: 10.1038/s41598-01701557-y

Garcia, J., de Gunzburg, J., Eychène, A., Gisselbrecht, S., and Porteu, F. (2001). Thrombopoietin-mediated sustained activation of extracellular signalregulated kinase in UT7-Mpl cells requires both Ras-Raf-1- and Rap1-B-Rafdependent pathways. Mol. Cell Biol. 21, 2659-2670. doi: 10.1128/MCB.21.8. 2659-2670.2001

Genin, M., Clement, F., Fattaccioli, A., Raes, M., and Michiels, C. (2015). M1 and M2 macrophages derived from THP-1 cells differentially modulate the response of cancer cells to etoposide. BMC Cancer 15:577. doi: 10.1186/s12885-0151546-9

Goulopoulou, S., McCarthy, C. G., and Webb, R. C. (2016). Toll-like receptors in the vascular system: sensing the dangers within. Pharmacol. Rev. 68, 142-167. doi: 10.1124/pr.114.010090

Gunderson, A. J., Mohammed, J., Horvath, F. J., Podolsky, M. A., Anderson, C. R., and Glick, A. B. (2013). CD8(+) T cells mediate RAS-induced psoriasislike skin inflammation through IFN- $\gamma$. J. Invest. Dermatol. 133, 955-963. doi: 10.1038/jid.2012.390

Guo, T., Wang, X., Qu, Y., Yin, Y., Jing, T., and Zhang, Q. (2015). Megakaryopoiesis and platelet production: insight into hematopoietic stem cell proliferation and differentiation. Stem Cell Investig. 2:3. doi: 10.3978/j.issn.2306-9759.2015. 02.01

Guzik, T. J., Korbut, R., and Adamek-Guzik, T. (2003). Nitric oxide and superoxide in inflammation and immune regulation. J. Physiol. Pharmacol. 54, 469-487.

Hall, B. M. (2015). T cells: soldiers and spies-the surveillance and control of effector T cells by regulatory T cells. Clin. J. Am. Soc. Nephrol. 10, 2050-2064. doi: 10.2215/CJN.06620714

Han, H. F., Zhang, L., Zhang, Y., Li, W. L., and Qu, H. B. (2014). Rapid quantification of total nitrogen and end-point determination of hide melting in manufacturing of donkey-hide gelatin. Zhongguo Zhong Yao Za Zhi 39, 1043-1047.

Harvey, K. A., Walker, C. L., Xu, Z., Whitley, P., Pavlina, T. M., Hise, M., et al. (2010). Oleic acid inhibits stearic acid-induced inhibition of cell growth and pro-inflammatory responses in human aortic endothelial cells. J. Lipid Res. 51, 3470-3480. doi: 10.1194/jlr.M010371

Hauling, T., Krautz, R., Markus, R., Volkenhoff, A., Kucerova, L., and Theopold, U. (2014). A Drosophila immune response against Ras-induced overgrowth. Biol. Open 3, 250-260. doi: 10.1242/bio.20146494

Heinemann, A., Schuligoi, R., Sabroe, I., Hartnell, A., and Peskar, B. A. (2003). Delta 12-prostaglandin J2, a plasma metabolite of prostaglandin D2, causes eosinophil mobilization from the bone marrow and primes eosinophils for chemotaxis. J. Immunol. 170, 4752-4758. doi: 10.4049/jimmunol.170.9.4752

Hitchcock, I. S., and Kaushansky, K. (2014). Thrombopoietin from beginning to end. Br. J. Haematol. 165, 259-268. doi: 10.1111/bjh.12772

Hopkins, A. L. (2008). Network pharmacology: the next paradigm in drug discovery. Nat. Chem. Biol. 4, 682-690. doi: 10.1038/nchembio.118 
Hou, Y., Nie, Y., Cheng, B., Tao, J., Ma, X., Jiang, M., et al. (2016). Qing fei Xiao yan Wan, a traditional Chinese medicine formula, ameliorates Pseudomonas aeruginosa-induced acute lung inflammation by regulation of PI3K/AKT and Ras/MAPK pathways. Acta Pharm. Sin. B 6, 212-221. doi: 10.1016/j.apsb.2016. 03.002

Ju, L., Wen, Y., Yin, J., Deng, S., Zheng, J., Wang, L., et al. (2016). Metabonomic study of the effects of different acupuncture directions on therapeutic efficacy. J. Chromatogr. B Analyt. Technol. Biomed. Life Sci. 1009-1010, 87-95. doi: 10.1016/j.jchromb.2015.12.006

Kakunaga, S., Ikeda, W., Shingai, T., Fujito, T., Yamada, A., Minami, Y., et al. (2004). Enhancement of serum- and platelet-derived growth factor-induced cell proliferation by Necl-5/Tage4/poliovirus receptor/CD155 through the RasRaf-MEK-ERK signaling. J. Biol. Chem. 279, 36419-36425. doi: 10.1074/jbc. M406340200

Kashiwagi, H., Yuhki, K., Kojima, F., Kumei, S., Takahata, O., Sakai, Y., et al. (2015). The novel prostaglandin I2 mimetic ONO-1301 escapes desensitization in an antiplatelet effect due to its inhibitory action on thromboxane A2 synthesis in mice. J. Pharmacol. Exp. Ther. 353, 269-278. doi: 10.1124/jpet.115.222612

Kim, S. M., Kim, C., Bae, H., Lee, J. H., Baek, S. H., Nam, D., et al. (2015). 6-Shogaol exerts anti-proliferative and pro-apoptotic effects through the modulation of STAT3 and MAPKs signaling pathways. Mol. Carcinog. 54, 1132-1146. doi: $10.1002 / \mathrm{mc} .22184$

Kitanaka, T., Nakano, R., Kitanaka, N., Kimura, T., Okabayashi, K., Narita, T., et al. (2017). JNK activation is essential for activation of MEK/ERK signaling in IL-1 $\beta$-induced COX-2 expression in synovial fibroblasts. Sci. Rep. 7:39914. doi: $10.1038 /$ srep39914

Kraakman, M. J., Lee, M. K., Al-Sharea, A., Dragoljevic, D., Barrett, T. J., Montenont, E., et al. (2017). Neutrophil-derived S100 calcium-binding proteins A8/A9 promote reticulated thrombocytosis and atherogenesis in diabetes. J. Clin. Invest. 127, 2133-2214. doi: 10.1172/JCI92450

Lembke, A., Gomez, R., Tenakoon, L., Keller, J., Cohen, G., Williams, G. H., et al. (2013). The mineralocorticoid receptor agonist, fludrocortisone, differentially inhibits pituitary-adrenal activity in humans with psychotic major depression. Psychoneuroendocrinology 38, 115-121. doi: 10.1016/j.psyneuen.2012.05.006

Li, X., Chen, C., An, Z. M., Li, Y. J., Zhang, M., He, H., et al. (2016). Effects of FGF-21 on osteogenic differentiation of human bone marrow mesenchymal stem cells in high glucose environment. Sichuan da xue xue bao yi xue ban 47 , 649-654.

Mao, Y., Hao, J., Jin, Z. Q., Niu, Y. Y., Yang, X., Liu, D., et al. (2017). Network pharmacology-based and clinically relevant prediction of the active ingredients and potential targets of Chinese herbs in metastatic breast cancer patients. Oncotarget 8, 27007-27021. doi: 10.18632/oncotarget.15351

Mazza, P., Minoia, C., Melpignano, A., Polimeno, G., Cascavilla, N., Di Renzo, N., et al. (2016). The use of thrombopoietin-receptor agonists (TPO-RAs) in immune thrombocytopenia (ITP): a "real life" retrospective multicenter experience of the Rete Ematologica Pugliese (REP). Ann. Hematol. 95, 239-244. doi: 10.1007/s00277-015-2556-z

Mazzanti, L., Cecati, M., Vignini, A., D’Eusanio, S., Emanuelli, M., Giannubilo, S. R., et al. (2011). Placental expression of endothelial and inducible nitric oxide synthase and nitric oxide levels in patients with HELLP syndrome. Am. J. Obstet. Gynecol. 205, 236.e1-236.e7. doi: 10.1016/j.ajog.2011. 04.022

Mejías, J. H., Lu, X., Osorio, C., Ullman, J. L., von Wettstein, D., and Rustgi, S. (2014). Analysis of wheat prolamins, the causative agents of celiac sprue, using reversed phase high performance liquid chromatography (RP-HPLC) and matrix-assisted laser desorption ionization time of flight mass spectrometry (MALDI-TOF-MS). Nutrients 6, 1578-1597. doi: 10.3390/nu6041578

Mendelson, K., Evans, T., and Hla, T. (2014). Sphingosine 1-phosphate signalling. Development 141, 5-9. doi: 10.1242/dev.094805

Morin-Poulard, I., Vincent, A., and Crozatier, M. (2013). The Drosophila JAKSTAT pathway in blood cell formation and immunity. JAKSTAT 2:e25700. doi: $10.4161 /$ jkst.25700

Murillo-Rodríguez, E., Giordano, M., Cabeza, R., Henriksen, S. J., Méndez, M., Navarro, L. et al. (2001). Oleamide modulates memory in rats. Neurosci. Lett. 313, 61-64. doi: 10.1016/S0304-3940(01)02256-X

Na, Y. R., Jung, D., Gu, G. J., and Seok, S. H. (2016). GM-CSF grown bone marrow derived cells are composed of phenotypically different dendritic cells and macrophages. Mol. Cells 39, 734-741. doi: 10.14348/molcells.2016.0160
Notarangelo, L. D. (2014). Immunodeficiency and immune dysregulation associated with proximal defects of $\mathrm{T}$ cell receptor signaling. Curr. Opin. Immunol. 31, 97-101. doi: 10.1016/j.coi.2014.10.003

Ohnishi, H., and Saito, Y. (2013). Eicosapentaenoic acid (EPA) reduces cardiovascular events: relationship with the EPA/arachidonic acid ratio. J. Atheroscler. Thromb. 20, 861-877. doi: 10.5551/jat.18002

Pang, H. Q., Yue, S. J., Tang, Y. P., Chen, Y. Y., Tan, Y. J., Cao, Y. J., et al. (2018). Integrated metabolomics and network pharmacology approach to explain possible action mechanisms of xin-sheng-hua granule for treating anemia. Front. Pharmacol. 9:165. doi: 10.3389/fphar.2018.00165

Peng, L., Li, J., Xu, Y., Wang, Y., Du, H., Shao, J., et al. (2016). The protective effect of beraprost sodium on diabetic nephropathy by inhibiting inflammation and p38 MAPK signaling pathway in high-fat diet/streptozotocin-induced diabetic rats. Int. J. Endocrinol. 2016:1690474. doi: 10.1155/2016/1690474

Queliza, K., Ihekweazu, F. D., Ali, A., and Kellermayer, R. (2017). Inflammatory bowel disease and immune thrombocytopenic purpura: combined immune dysregulation in an adolescent. Ann. Clin. Lab. Sci. 47, 226-228.

Raber, P. L., Thevenot, P., Sierra, R., Wyczechowska, D., Halle, D., Ramirez, M. E., et al. (2014). Subpopulations of myeloid-derived suppressor cells impair T cell responses through independent nitric oxide-related pathways. Int. J. Cancer 134, 2853-2864. doi: $10.1002 / \mathrm{ijc} .28622$

Rajalingam, R. (2016). The impact of HLA class i-specific killer cell immunoglobulin-like receptors on antibody-dependent natural killer cellmediated cytotoxicity and organ allograft rejection. Front. Immunol. 7:585. doi: 10.3389/fimmu.2016.00585

Ramakrishnan, S., Anand, V., and Roy, S. (2014). Vascular endothelial growth factor signaling in hypoxia and inflammation. J. Neuroimmune Pharmacol. 9, 142-160. doi: 10.1007/s11481-014-9531-7

Roche, P. A., and Furuta, K. (2015). The ins and outs of MHC class IImediated antigen processing and presentation. Nat. Rev. Immunol. 15, 203-216. doi: $10.1038 / \mathrm{nri3} 818$

Rodeghiero, F., Stasi, R., Gernsheimer, T., Michel, M., Provan, D., Arnold, D. M., et al. (2009). Standardization of terminology, definitions and outcome criteria in immune thrombocytopenic purpura of adults and children: report from an international working group. Blood 113, 2386-2393. doi: 10.1182/blood-2008$07-162503$

Roy, J., Adili, R., Kulmacz, R., Holinstat, M., and Das, A. (2016). Development of poly unsaturated fatty acid derivatives of aspirin for inhibition of platelet function. J. Pharmacol. Exp. Ther. 359, 134-141. doi: 10.1124/jpet.116.234781

Roy, S., Guler, R., Parihar, S. P., Schmeier, S., Kaczkowski, B., Nishimura, H., et al. (2015). Batf2/Irf1 induces inflammatory responses in classically activated macrophages, lipopolysaccharides, and mycobacterial infection. J. Immunol. 194, 6035-6044. doi: 10.4049/jimmunol.1402521

Sahoo, A., Alekseev, A., Tanaka, K., Obertas, L., Lerman, B., Haymaker, C., et al. (2015). Batf is important for IL-4 expression in T follicular helper cells. Nat. Commun. 6:7997. doi: 10.1038/ncomms8997

Schmitz, P. G., Zhang, K., and Dalal, R. (2000). Eicosapentaenoic acid suppresses PDGF-induced DNA synthesis in rat mesangial cells: involvement of thromboxane A2. Kidney Int. 57, 1041-1051. doi: 10.1038/sj.ki.449 1425

Schuck, R. N., Zha, W., Edin, M. L., Gruzdev, A., Vendrov, K. C., Miller, T. M., et al. (2014). The cytochrome P450 epoxygenase pathway regulates the hepatic inflammatory response in fatty liver disease. PLoS One 9:e110162. doi: 10.1371/ journal.pone. 0110162

Shajahan-Haq, A. N., Cheema, M. S., and Clarke, R. (2015). Application of metabolomics in drug resistant breast cancer research. Metabolites 5, 100-118. doi: 10.3390/metabo5010100

Starkie, R., Ostrowski, S. R., Jauffred, S., Febbraio, M., and Pedersen, B. K. (2003). Exercise and IL-6 infusion inhibit endotoxin-induced TNF-alpha production in humans. FASEB J. 17, 884-886. doi: 10.1096/fj.02-0670fje

Swain, T. R., Jena, R. K., and Swain, K. P. (2016). High dose intravenous anti$\mathrm{D}$ immune globulin is more effective and safe in Indian paediatric patients of immune thrombocytopenic purpura. J. Clin. Diagn. Res. 10, FC12-FC15. doi: $10.7860 / J C D R / 2016 / 20347.8976$

Talaat, R. M., Elmaghraby, A. M., Barakat, S. S., and El-Shahat, M. (2014). Alterations in immune cell subsets and their cytokine secretion profile in childhood idiopathic thrombocytopenic purpura (ITP). Clin. Exp. Immunol. 176, 291-300. doi: 10.1111/cei.12279 
van Diepen, J. A., Berbée, J. F., Havekes, L. M., and Rensen, P. C. (2013). Interactions between inflammation and lipid metabolism: relevance for efficacy of anti-inflammatory drugs in the treatment of atherosclerosis. Atherosclerosis 228, 306-315. doi: 10.1016/j.atherosclerosis.2013.02.028

Wagner, J., Granvogl, M., and Schieberle, P. (2016). Characterization of the key aroma compounds in raw licorice (Glycyrrhiza glabra L.) by means of molecular sensory science. J. Agric. Food Chem. 64, 8388-8396. doi: 10.1021/acs.jafc. $6 \mathrm{~b} 03676$

Walsh, K. B., Teijaro, J. R., Brock, L. G., Fremgen, D. M., Collins, P. L., Rosen, H., et al. (2014). Animal model of respiratory syncytial virus: CD8 $+\mathrm{T}$ cells cause a cytokine storm that is chemically tractable by sphingosine-1-phosphate 1 receptor agonist therapy. J. Virol. 88, 6281-6293. doi: 10.1128/JVI.004 64-14

Wang, W., Graeler, M. H., and Goetzl, E. J. (2005). Type 4 sphingosine 1-phosphate $\mathrm{G}$ protein-coupled receptor (S1P4) transduces S1P effects on T cell proliferation and cytokine secretion without signaling migration. FASEB J. 19, 1731-1733. doi: 10.1096/fj.05-3730fje

Yang, Y. F., Zhou, A. X., and Ma, R. (1994). Stablishment of animal model of immune thrombocytopenic purple. Chin. Hematol. 15, 160-161.

Yao, R., Lin, Y., Li, Q., Zhou, X., Pan, X., Bao, Y., et al. (2014). Downregulation of T-bet/GATA-3 ratio induced by IL-11 treatment is responsible for Th1/Th2 balance restoration in human immune thrombocytopenic purpura (ITP). J. Thromb. Thrombol. 38, 183-189. doi: 10.1007/s11239-0131036-3

Zhang, L., Chen, K., Li, T., He, H., Hou, L., Wu, X., et al. (2016). Tian $\mathrm{S}$ Prednison provokes serum and vasoactive substances in a mice model of immune thrombocytopenia. Iran. J. Basic Med. Sci. 19, 1010-1015. doi: 10.22038/ijbms.2016.7602

Zhong, Y., Byrd, J. C., and Dubovsky, J. A. (2014). The B-cell receptor pathway: a critical component of healthy and malignant immune biology. Semin. Hematol. 51, 206-218. doi: 10.1053/j.seminhematol.2014.05.007

Zhou, X., Li, Y., Peng, Y., Hu, J., Zhang, R., He, L., et al. (2014). Clinical phenotype network: the underlying mechanism for personalized diagnosis and treatment of traditional chinese medicine. Front. Med. 8, 337-346. doi: 10.1007/s11684014-0349-8

Conflict of Interest Statement: WL and HL was employed by Tasly Pharmaceutical Group.

The remaining authors declare that the research was conducted in the absence of any commercial or financial relationships that could be construed as a potential conflict of interest.

The reviewer CF and handling Editor declared their shared affiliation.

Copyright (C) $2018 \mathrm{Li}, \mathrm{Li}, \mathrm{Lu}, \mathrm{Li}$, Wang, Luo, Wu, Dong, Bai and Zhang. This is an open-access article distributed under the terms of the Creative Commons Attribution License (CC BY). The use, distribution or reproduction in other forums is permitted, provided the original author(s) and the copyright owner are credited and that the original publication in this journal is cited, in accordance with accepted academic practice. No use, distribution or reproduction is permitted which does not comply with these terms. 